\title{
Learning and sharing at the Lausanne 2020 Youth Olympic Games medical clinic
}

\section{EDUCATION / INNOVATION / SPORTS PHYSIOTHERAPY / SPORTS SCIENCE / TEAM MANAGEMENT}

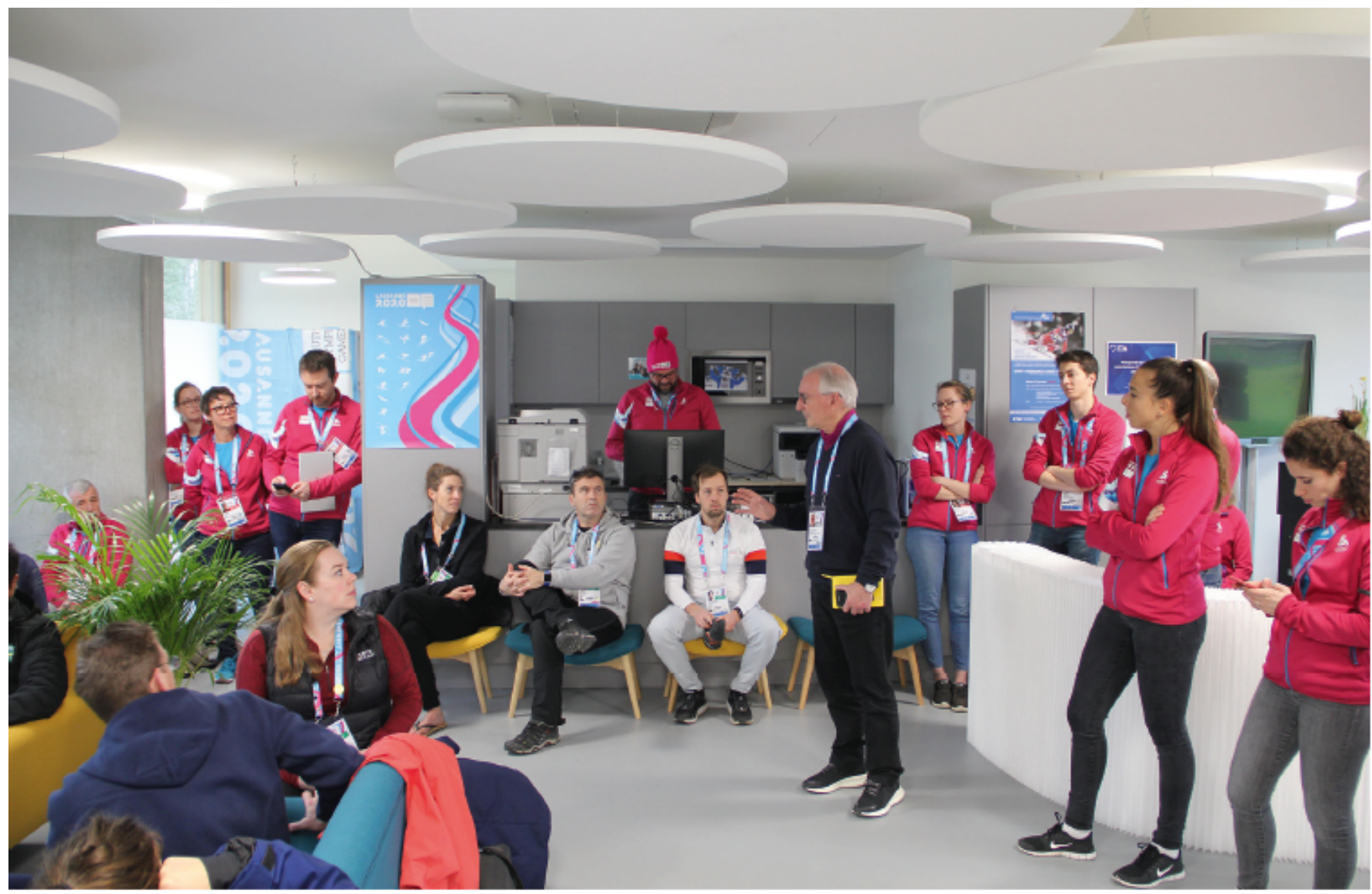

\section{Gard Suzanne}

Interdisciplinary Division for Adolescent Health (DISA), Department of Women-Mother-Child, University Hospital of Lausanne, Lausanne, Switzerland

\section{The concept}

The Youth Olympic Games are an elite sporting event that include a series of educational activities and we built the whole concept of medical care at the village in the same spirit. We wanted not only to build a clinic with a lot of expertise, but to create a space for education for the young athletes, their medical teams and our volunteers.

We recruited 3 levels of volunteers: experienced in their field of sports medicine, young professionals and 
students. Altogether, we had 109 professionals and 40 students (medicine, nursing and physiotherapy). The latter were at the front with the young athletes, supervised and mentored by experienced professionals. The medical students added value to the care of the athletes by accompanying them for necessary transfers to the main hospital for imaging or surgery, thereby enhancing continuity between the Village clinic and the hospital. Physiotherapy students were in charge of the passive recovery zone, with massages and recovery pressure boots, facilitation connection with the young athletes. Nursing students welcomed patients for the initial care and following the cases we kept for surveillance, always under supervision. In addition, all students were active in the flash information and demonstration campaigns throughout the Village to prevent the spreading of the seasonal flu. They were brilliant in conveying the importance of hand sanitization. These educational actions made by the young, for the young, became quite popular and, considering the eventual turn of events (COVID-19), turned out to be very important messages.

\section{CPD workshops in the clinic}

To make the most of the time around the care for the athletes, we encouraged Continuing Professional Development (CPD) for our staff. We encouraged our volunteers to prepare topics they could teach in an informal way during the games. Eventually, they delivered 41 spontaneous workshops, during the quieter times of the clinic, where they shared their skills and knowledge. Almost all of them were given by a multidisciplinary team and many topics were covered, e.g. ankle sprains, how to use an epinephrine pen, ultrasound diagnosis for the shoulder, sports osteopathy or podiatry, case studies and many more. These workshops gave everyone the opportunity to discover the different point of views, as well as the skills and knowledge of other health professions.

\section{The Academic Programme Lausanne2020}

Twice a day during the Games, the waiting room of the Medical Clinic transformed into a vibrant conference lounge. The Academic Programme was initially created for the volunteers of the clinic, but together with Dr Stéphane Tercier (clinic director), we wanted to add value for other members of the YOG delegations.

The short format of the conferences (30 minutes) and the schedule (10 am and $4 \mathrm{pm}$ ) were designed in the hope of attracting National Olympic Committee (NOC) medical staff members to join. The topics were intentionally very diverse (e.g. paediatric ACL injuries, concussion in sports management, dental care in athletes or abuse in sports), and the speakers were a good mix of clinicians, researchers, medical students and athletes. We repeated most of the talks twice to give the opportunity to a greater number to attend each topic.

The attendance surpassed our expectations, by the number and diversity: 20 volunteer staff: doctors, physios, nurses, dentists, podiatrists, osteopaths, chiropractors, pharmacists, administrative staff and students along with an average of 12 NOC medical team members. The mean participation of the 56 NOC medical team members was 3.9 conferences during their stay at the Olympic Village.

Dr Richard Budgett (IOC Medical director) and Prof. Lars Engebretsen (IOC Head of Science and Research) supported and approved the programme officially. This allowed us to attribute credits for each participant, provided they attended at least three presentations. They received a Certificate of attendance, signed by the IOC medical board members. 


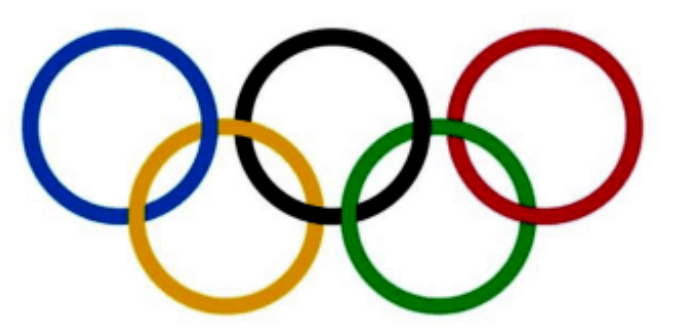

INTERNATIONAL OLYMPIC COMMITTEE

\author{
LAUSANNE2020 ACADEMIC PROGRAMME \\ SPORTS MEDICINE CONFERENCES IN THE MEDICAL CLINIC, VORTEX
}

From January $9^{\text {th }}$ to January $22^{\text {nd, }} 2020$

\title{
CERTIFICATE
}

\author{
John Doe
}

has attended conferences on:

\begin{tabular}{ll}
\hline Consensus on concussion for RTP & Dr Boris Gojanovic \\
\hline The role of the IOC in protecting athlete health & Dr Richard Budgett \\
\hline IOC ACL paediatric consensus statement & Prof. Lars Engebretsen \\
\hline Artistic sport challenges & Yoonmi Lehmann, athlete \\
\hline Chiropraxis in sports & Dr Roland Noirat, chiropractitioner \\
\hline Overtraining syndrome: easy to miss & Dr Justin Carrard \\
\hline Dental care in athletes: why is it important? & Prof. Martin Broome \\
\hline Ankle sprain rehabilitation: important matter! & Aline Reinmann, PT, MSc \\
\hline Sports psychology: how can it help? & Mélanie Hindi, sports psychologist \\
\hline Osteochondral lesions: how do we deal? & Dr Benjamin Tschopp \\
\hline ECG in athletes & Dr Karsten Königstein \\
\hline Taking care of adolescent patients & Dr Takeuchi Yusuke \\
\hline Abuse in sports be aware! & Dr Anne-Emmanuelle Ambresin \\
\hline Pregnancy and return to sport & Dr Silvia Bonfanti \\
\hline Scapular dyskinesis in sports: an update & Gard Suzanne, PT, MSc \\
\hline Diagnostic ultrasound in sports medicine & Dr Maximilian Schindler \\
\hline Asthma in endurance sports & Damien Choffat and Nicolas Domon, Med students \\
\hline
\end{tabular}

Scientific and organization committee: Suzanne Gard and Stéphane Tercier

Total: 8.5 hours of CPD

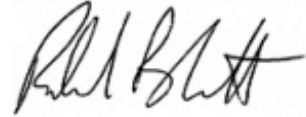

Richard Budgett

IOC Medical and Scientific Director

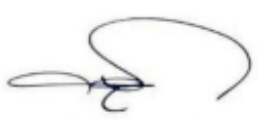

Ugur Erdener

IOC Medical and Scientific Commission Chairperson

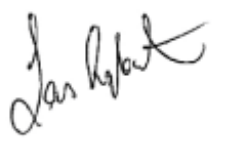

Lars Engebretsen IOC Head of Scientific Activities 


\section{Sharing and connecting}

This routine twice a day at the same time gave us the opportunity to create links with the NOC teams and most important gave us the opportunity to discuss, exchange point of views in a safe place around these topics. As the days went by, some of them would often come and whenever they came with an injured athlete, it made things easier because the connection had been established.

The Academic Programme Lausanne2020 gave us the opportunity not only to contribute to the CPD for our volunteers, but also to create connections and exchanges with the NOC teams from across the world. It gave us a sense of community in this field of sports medicine, all sharing the same goals to protect the athletes' health and promote a safe participation at the Youth Olympic Games.

Finally, through the workshops, the academic programme and the care we provided to athletes, we learned more about the benefits of working in a multidisciplinary team, with a flat hierarchy across professions; it is really very efficient and fulfilling, and we all concluded that we wish we could always work like this. \#DREAMCLINIC.

\section{Corresponding author}

Suzanne Gard, PT, MSc

Deputy Venue Medical Manager

YOV medical clinic Lausanne2020

suzanne.gard@chuv.ch

Twitter: @SuzanneGard

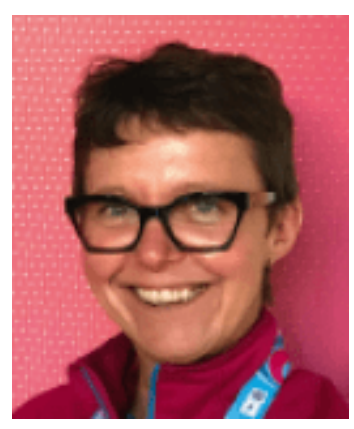

